\title{
Extension of Wolfe Method for Solving Quadratic Programming with Interval Coefficients
}

\author{
Syaripuddin, ${ }^{1}$ Herry Suprajitno, ${ }^{2}$ and Fatmawati ${ }^{2}$ \\ ${ }^{1}$ Department of Mathematics, Faculty of Mathematics and Natural Sciences, Mulawarman University, \\ Kampus Gunung Kelua, Jl. Barong Tongkok, Samarinda 1068, Indonesia \\ ${ }^{2}$ Department of Mathematics, Faculty of Science and Technology, Airlangga University, Kampus C Unair, \\ Jl. Mulyorejo, Surabaya 60115, Indonesia
}

Correspondence should be addressed to Fatmawati; fatma47unair@gmail.com

Received 10 April 2017; Accepted 1 August 2017; Published 14 September 2017

Academic Editor: Frank Werner

Copyright (C) 2017 Syaripuddin et al. This is an open access article distributed under the Creative Commons Attribution License, which permits unrestricted use, distribution, and reproduction in any medium, provided the original work is properly cited.

Quadratic programming with interval coefficients developed to overcome cases in classic quadratic programming where the coefficient value is unknown and must be estimated. This paper discusses the extension of Wolfe method. The extended Wolfe method can be used to solve quadratic programming with interval coefficients. The extension process of Wolfe method involves the transformation of the quadratic programming with interval coefficients model into linear programming with interval coefficients model. The next step is transforming linear programming with interval coefficients model into two classic linear programming models with special characteristics, namely, the optimum best and the worst optimum problem.

\section{Introduction}

Quadratic programming is a special form of nonlinear programming which has special characteristics; that is, the objective function is in quadratic forms and constraint functions are linear form [1]. Although the quadratic programming is part of nonlinear programming, the completion is still adopting some linear programming problem solving methods, one of which is the Wolfe method. This method transforms the quadratic programming problem into a linear programming problem. Wolfe [2] modified the simplex method to solve quadratic programming problem by adding conditions of the Karush-Kuhn-Tucker (KKT) and changing the objective function of quadratic forms into a linear form.

Interval quadratic programming is a development of the classic quadratic programming that utilizes interval analysis theory developed by Moore [3]. This development aim is to accommodate cases which contain the uncertainty, that is, when the data value is unknown for certain, but the data lies within an interval where the values of the upper limit and lower limit are known. The special characteristics of the interval quadratic programming problem are the coefficients of the objective function and constraint functions are in the interval form.

Research on quadratic programming with interval coefficients has been conducted by Liu and Wang [4]. However, the coefficients of quadratic forms in the objective function on the developed model are not in the interval form yet. Furthermore, Li and Tian [5] generalized the model in [4] by assuming that the quadratic coefficients of the objective function are in the interval form. References $[4,5]$ used the duality theory to create a method of solving the quadratic programming with interval coefficients. Quadratic programming model with interval coefficients is transformed into two classic quadratic programming models with the special characteristics, called the best optimum and worst optimum problem. A completion method was developed based on the method of solving the linear programming with interval coefficients that have been discussed by some researchers [6$10]$.

This paper will discuss the extension of Wolfe method to solve quadratic programming with interval coefficients. Therefore, this article will focus on how to transform the quadratic programming with interval coefficients into linear 
programming with interval coefficients. Furthermore, the linear programming with interval coefficients which has been obtained from the transformation will be solved by using the method in [8].

This paper is organized as follows. Section 2 discusses interval arithmetic operations. In Section 3, a general form of linear programming with interval coefficients is stated. In Section 4, a general form of quadratic programming with interval coefficients is stated. Extension of Wolfe method as one method of solving the quadratic programming with interval coefficients is discussed in Section 5, whereas Section 6 discusses numerical examples, and Section 7 provides some concluding remarks.

\section{Interval Arithmetic}

The basic definition and properties of interval number and interval arithmetic can be seen at Moore [3], Alefeld and Herzberg [11], and Hansen [12].

Definition 1. A closed real interval $\underline{x}=\left[x_{I}, x_{S}\right]$ denoted by $\underline{x}$ is a real interval number which can be defined completely by

$$
\underline{x}=\left[x_{I}, x_{S}\right]=\left\{x \in \mathfrak{R} \mid x_{I} \leq x \leq x_{S} ; x_{I}, x_{S} \in \mathfrak{R}\right\},
$$

where $x_{I}$ and $x_{S}$ are called infimum and supremum, respectively.

Definition 2. A real interval number $\underline{x}=\left[x_{I}, x_{S}\right]$ is called degenerate, if $x_{I}=x_{S}$.

Definition 3. Let $\underline{x}=\left[x_{I}, x_{S}\right]$ and $\underline{y}=\left[y_{I}, y_{S}\right]$; then

1. $\underline{x}+\underline{y}=\left[x_{I}+y_{I}, x_{S}+y_{S}\right]$ (addition),

2. $\underline{x}-\underline{y}=\left[x_{I}, x_{S}\right]-\left[y_{I}, y_{S}\right]=\left[x_{I}, x_{S}\right]+\left[-y_{S},-y_{I}\right]=$ $\left[x_{I}-y_{S}, x_{S}-y_{I}\right]$ (subtraction),

3. $\underline{x} \cdot \underline{y}=\left[\min \left\{x_{I} y_{I}, x_{I} y_{S}, x_{S} y_{I}, x_{S} y_{S}\right\}, \max \left\{x_{I} y_{I}, x_{I} y_{S}\right.\right.$, $\left.\left.x_{S} y_{I}, x_{S} y_{S}\right\}\right]$ (multiplication),

4. $\underline{x} / \underline{y}=\underline{x}(1 / \underline{y})=\left[x_{I}, x_{S}\right]\left[1 / y_{S}, 1 / y_{I}\right], 0 \notin \underline{y}$ (division).

\section{Linear Programming with Interval Coefficients}

The general form of linear programming with interval coefficients is defined as follows:

$$
\text { Maximize } \quad \underline{z}=\sum_{j=1}^{n}\left[c_{j I}, c_{j S}\right] x_{j}
$$

subject to

$$
\begin{gathered}
\sum_{j=1}^{n}\left[a_{i j I}, a_{i j S}\right] x_{j} \leq\left[b_{i I}, b_{i S}\right], \quad i=1,2, \ldots, m \\
x_{j} \geq 0, \quad j=1,2, \ldots, n,
\end{gathered}
$$

where $x_{j} \in \mathfrak{R},\left[c_{j I}, c_{j S}\right],\left[b_{i I}, b_{i S}\right]$, and $\left[a_{i j I}, a_{i j S}\right] \in I(\mathfrak{R})$.
The model in $(2 a)-(2 c)$ is solved by means of transforming the linear programming with interval coefficients into two classic linear programming models with the special characteristics, namely, the best optimum and the worst optimum problems. The best optimum problem has properties of best version on the objective function and maximum feasible area on the constraint function. On the other hand, the worst optimum problem has a characteristic that it is the worst version of the objective function and the minimum feasible area of the constraint function.

Chinneck and Ramadan [8] provide a rule to determine the best and worst optimum problem in a linear programming problem with interval coefficients. The constraints of linear programming with interval coefficients which have an inequality sign $(\leq)$ in $(2 \mathrm{~b})$ have characteristics of the maximum feasible area and the minimum feasible area which is given by the following theorem.

Theorem 4 (Chinneck and Ramadan [8]). Suppose that we have an interval inequality given $\sum_{j=1}^{n}\left[a_{j I}, a_{j S}\right] x_{j} \leq\left[b_{I}, b_{S}\right]$, where $x_{j} \geq 0$. Then, $\sum_{j=1}^{n} a_{j I} x_{j} \leq b_{S}$ is maximum feasible area and $\sum_{j=1}^{n} a_{j S} x_{j} \leq b_{I}$ is minimum feasible area.

The objective function of linear programming with interval coefficients for the case of maximizing (2a) has characteristics of the best version and the worst version of the objective function is expressed in the following theorem.

Theorem 5 (Chinneck and Ramadan [8]). If $\underline{z}=\sum_{j=1}^{n}\left[c_{j I}\right.$, $\left.c_{j S}\right] x_{j}$ is the objective function for $x_{j} \geq 0$, then $\sum_{j=1}^{n} c_{j S} x_{j} \geq$ $\sum_{j=1}^{n} c_{j I} x_{j}$, where $\sum_{j=1}^{n} c_{j S} x_{j}$ is the best version of the objective function and $\sum_{j=1}^{n} c_{j I} x_{j}$ is the worst version of the objective function.

\section{Quadratic Programming with Interval Coefficients}

The general form of quadratic programming with interval coefficients introduced by $\mathrm{Li}$ and Tian [5] is defined as follows:

$$
\begin{aligned}
& \text { Maximize } \underline{\underline{z}} \\
& \qquad \sum_{j=1}^{n}\left[c_{j I}, c_{j S}\right] x_{j} \\
& +\frac{1}{2} \sum_{j=1}^{n} \sum_{k=1}^{n}\left[q_{j k I}, q_{j k S}\right] x_{j} x_{k}
\end{aligned}
$$

subject to

$$
\begin{gathered}
\sum_{j=1}^{n}\left[a_{i j I}, a_{i j S}\right] x_{j} \leq\left[b_{i I}, b_{i S}\right], \quad i=1,2, \ldots, m \\
x_{j} \geq 0, \quad j=1,2, \ldots, n,
\end{gathered}
$$


where $x_{j} \in \Re,\left[c_{j I}, c_{j S}\right],\left[q_{i j I}, q_{i j S}\right],\left[b_{i I}, b_{i S}\right],\left[a_{i j I}, a_{i j S}\right] \in I(\Re)$, $\sum_{j=1}^{n} \sum_{k=1}^{n}\left[q_{j k I}, q_{j k s}\right] x_{j} x_{k}$ is negative semidefinite, and $I(\Re)$ are the set of all interval numbers in $\mathfrak{R}$.

The model as shown in (3a)-(3c) is a generalization of the model in [4]. The coefficient of the objective function and constraints of the quadratic programming with interval coefficients model in (3a)-(3c) have an interval form. The idea to solve the model is the extension of Wolfe method. This method focuses on how to transform the quadratic programming with interval coefficients in (3a)-(3c) into linear programming with interval coefficients in (2a)-(2c). Furthermore, linear programming with interval coefficients obtained from the transformation is done using the method in [8].

Extension of Wolfe method is the main result in this paper. The fundamental difference between the extensions of Wolfe method and the method in [5] is, on the extension of Wolfe method, quadratic programming model with interval coefficients is transformed into linear programming with interval coefficients, while, in [5], the model of quadratic programming with interval coefficients is maintained.

\section{Extension of Wolfe Method}

Wolfe method is one method for solving quadratic programming problems by means of transforming the quadratic programming problems into a linear programming problem. Wolfe [2] modified the simplex method to solve quadratic programming problems by adding a requirement KarushKuhn-Tucker (KKT) and changing the quadratic objective function into a linear objective function.

The extension of Wolfe method is used to solve quadratic programming problem with interval coefficients. Steps of extension of Wolfe method are declared as follows. is

Form of Lagrange function for the problem in (3a)-(3c)

$$
\begin{aligned}
& L(x, y, r, \lambda, \mu)=\sum_{j=1}^{n}\left[c_{j I}, c_{j S}\right] x_{j}+\frac{1}{2} \\
& \quad \cdot \sum_{j=1}^{n} \sum_{k=1}^{n}\left[q_{j k I}, q_{j k S}\right] x_{j} x_{k} \\
& \quad-\sum_{i=1}^{m} \lambda_{i}\left(\sum_{j=1}^{n}\left(\left[a_{i j I}, a_{i j S}\right] x_{j}-\left[b_{i I}, b_{i S}\right]+y_{i}^{2}\right)\right) \\
& \quad-\sum_{j=1}^{n} \mu_{j}\left(-x_{j}+r_{j}^{2}\right),
\end{aligned}
$$

where $\lambda_{i}, i=1,2, \ldots, m, \mu_{j}, j=1,2, \ldots, n$, are Lagrange multipliers and $L(x, y, r, \lambda, \mu)$ is Lagrange function with interval coefficients.

Local minimum points of the function $L$ were obtained by the first partial derivatives of the function $L$ with respect to the variables and equating to zero (KKT necessary conditions) (see $[13,14])$.

$$
\begin{gathered}
\frac{\partial L}{\partial x_{i}}=\left[c_{j I}, c_{j S}\right]+\sum_{k=1}^{n}\left[q_{j k I}, q_{j k S}\right] x_{k} \\
\quad-\sum_{i=1}^{m}\left[a_{i j I}, a_{i j S}\right] \lambda_{i}+\mu_{j}=0, \\
j=1,2, \ldots, n, \\
\frac{\partial L}{\partial y_{i}}=-2 \lambda_{i} y_{i}=0, \quad i=1,2, \ldots, m, \\
\frac{\partial L}{\partial r_{i}}=-2 \mu_{j} r_{j}=0, \quad j=1,2, \ldots, n, \\
\frac{\partial L}{\partial \lambda_{i}}=\sum_{j=1}^{n}\left[a_{i j I}, a_{i j s}\right] x_{j}+y_{i}^{2}-\left[b_{i I}, b_{i S}\right] \\
=0, \quad i=1,2, \ldots, m, \\
\frac{\partial L}{\partial \mu_{i}}=x_{j}-r_{j}^{2}=0, \quad j=1,2, \ldots, n, \\
x_{j}, \lambda_{i}, \mu_{j}, y_{i}, r_{j} \geq 0, \quad i=1,2, \ldots, m, \quad j=1,2, \ldots, n .
\end{gathered}
$$

Results simplification of (5a)-(5f) is

$$
\begin{aligned}
& -\sum_{k=1}^{n}\left[q_{j k I}, q_{j k s}\right] x_{k}+\sum_{i=1}^{m}\left[a_{i j I}, a_{i j S}\right] \lambda_{i}-\mu_{j} \\
& \quad=\left[c_{j I}, c_{j S}\right], \quad j=1,2, \ldots, n, \\
& \sum_{j=1}^{n}\left[a_{i j I}, a_{i j s}\right] x_{j}+s_{i}=\left[b_{i I}, b_{i S}\right], \quad i=1,2, \ldots, m, \\
& x_{j}, \lambda_{i}, \mu_{j}, s_{i} \geq 0, \quad j=1,2, \ldots, n, i=1,2, \ldots, m
\end{aligned}
$$

and satisfies the complementary conditions,

$$
\mu_{j} x_{j}=0, \quad j=1,2, \ldots, n, \lambda_{i} s_{i}=0, i=1,2, \ldots, m .
$$

Add artificial variables $v_{j}, j=1,2, \ldots, n$, in (6a) for an initial basis, as follows:

$$
\begin{aligned}
& -\sum_{k=1}^{n}\left[q_{j k I}, q_{j k S}\right] x_{k}+\sum_{i=1}^{m}\left[a_{i j I}, a_{i j S}\right] \lambda_{i}-\mu_{j}+v_{j} \\
& \quad=\left[c_{j I}, c_{j S}\right] .
\end{aligned}
$$

Furthermore, create a linear programming with interval coefficients, where the objective function is to minimize the number of artificial variables $v_{j}, j=1,2, \ldots, n$, and constraint is (7), (6b), (6c), and (6d) obtained from necessary conditions of KKT.

$$
\text { Minimize } \underline{z}=v_{1}+v_{2}+\cdots+v_{n}
$$


subject to,

$$
\begin{aligned}
& -\sum_{k=1}^{n}\left[q_{j k I}, q_{j k S}\right] x_{k}+\sum_{i=1}^{m}\left[a_{i j I}, a_{i j S}\right] \lambda_{i}-\mu_{j}+v_{j} \\
& =\left[c_{j I}, c_{j S}\right], \quad j=1,2, \ldots, n \\
& \sum_{j=1}^{n}\left[a_{i j I}, a_{i j s}\right] x_{j}+s_{i}=\left[b_{i I}, b_{i S}\right], \quad i=1,2, \ldots, m \\
& x_{j}, \lambda_{i}, \mu_{j}, s_{i}, v_{j} \geq 0, \quad j=1,2, \ldots, n, i=1,2, \ldots, m
\end{aligned}
$$

satisfying the complementary conditions,

$$
\begin{aligned}
\mu_{j} x_{j} & =0, \quad j=1,2, \ldots, n, \\
\lambda_{i} s_{i} & =0, \quad i=1,2, \ldots, m,
\end{aligned}
$$

where $v_{j} \geq 0$ is artificial variable.

The model as shown in (8a)-(8e) is linear programming with interval coefficients which is added by complementary conditions. This model is the result of the transformation from the quadratic programming with interval coefficients model by extension of Wolfe method.

The next step, linear programming with interval coefficients model in (8a)-(8e), was solved by transforming into two linear programming cases with the special characteristics, namely, the best and the worst optimum problem. The transformation process can be written in Algorithm 6 as follows.

Algorithm 6.

(1) Given a quadratic programming problem with interval coefficients in (3a)-(3c), Extension of Wolfe method is based on $(3 a)-(3 c)$ equivalent to the linear programming with interval coefficients in (8a)-(8e).

(2) Use Theorems 4 and 5 for transforming the linear programming with interval coefficients in (8a)-(8e) into two classic linear programming models with special characteristics; namely,

(a) the best optimum problem is

$$
\text { Minimize } z_{S}=v_{1}+v_{2}+\cdots+v_{n}
$$

subject to,

$$
-\sum_{k=1}^{n} q_{j k S} x_{k}+\sum_{i=1}^{m} a_{i j I} \lambda_{i}-\mu_{j}+v_{j}=c_{j S},
$$

$$
j=1,2, \ldots, n
$$$$
\sum_{j=1}^{n} a_{i j I} x_{j}+s_{i}=b_{i S}
$$

$$
i=1,2, \ldots, m
$$

$$
\begin{aligned}
x_{j}, \lambda_{i}, \mu_{j}, s_{i}, v_{j} \geq 0, \\
\quad j=1,2, \ldots, n, i=1,2, \ldots, m
\end{aligned}
$$

satisfying the complementary conditions,

$$
\begin{aligned}
\mu_{j} x_{j} & =0, \quad j=1,2, \ldots, n, \\
\lambda_{i} s_{i} & =0, \quad i=1,2, \ldots, m,
\end{aligned}
$$

(b) the worst optimum problem is

$$
\begin{aligned}
& \text { Minimize } z_{I}=v_{1}+v_{2}+\cdots+v_{n} \\
& \text { subject to, } \\
& -\sum_{k=1}^{n} q_{j k I} x_{k}+\sum_{i=1}^{m} a_{i j S} \lambda_{i}-\mu_{j}+v_{j}=c_{j I}, \\
& \qquad \begin{array}{l}
\sum_{j=1}^{n} a_{i j S} x_{j}+s_{i}=b_{i I}, \\
\quad i=1,2, \ldots, n \\
x_{j}, \lambda_{i}, \mu_{j}, s_{i}, v_{j} \geq 0, \\
j=1,2, \ldots, n, \quad i=1,2, \ldots, m
\end{array}
\end{aligned}
$$

satisfying the complementary conditions,

$$
\begin{aligned}
\mu_{j} x_{j} & =0, \quad j=1,2, \ldots, n, \\
\lambda_{i} s_{i} & =0, \quad i=1,2, \ldots, m .
\end{aligned}
$$

(3) The optimum value of the quadratic programming with interval coefficients is obtained by combining the optimum value from the worst and the best optimum problem; that is, $\underline{z}=\left[z_{I}, z_{S}\right]$.

Algorithm 6 shows that the best and the worst optimum problem are linear programming models added by complementary conditions. Thus, both problems can be solved by simplex method.

\section{Numerical Example}

Consider the following example of quadratic programming with interval coefficients in the journal Li and Tian [5].

$$
\begin{aligned}
\text { Minimize } & z \\
& =[-10,-6] x_{1}+[2,3] x_{2}+[-1,1] x_{1} x_{2} \\
& \quad+[4,10] x_{1}^{2}+[10,20] x_{2}^{2}
\end{aligned}
$$

subject to

$$
\begin{aligned}
{[1,2] x_{1}+3 x_{2} } & \leq[1,10] \\
{[-2,8] x_{1}+[4,6] x_{2} } & \leq[4,6] \\
x_{1}, x_{2} & \geq 0 .
\end{aligned}
$$


TABLE 1: Two classic linear programming models with special characteristics.

\begin{tabular}{ll}
\hline The best optimum problem & The worst optimum problem \\
\hline (1) Classic linear programming model & (2) Classic linear programming model \\
Minimize $\quad z_{S}=v_{1}+v_{2}$ & Minimize $z_{I}=v_{1}+v_{2}$ \\
subject to & subject to \\
$8 x_{1}-x_{2}+\lambda_{1}-2 \lambda_{2}-\mu_{1}+v_{1}=10$ & $20 x_{1}+x_{2}+2 \lambda_{1}+8 \lambda_{2}-\mu_{1}+v_{1}=6$ \\
$x_{1}-20 x_{2}-3 \lambda_{1}-4 \lambda_{2}+\mu_{2}+v_{2}=2$ & $-x_{1}-40 x_{2}-3 \lambda_{1}-6 \lambda_{2}+\mu_{2}+v_{2}=3$ \\
$x_{1}+3 x_{2}+s_{1}=10$ & $2 x_{1}+3 x_{2}+s_{1}=1$ \\
$-2 x_{1}+4 x_{2}+s_{2}=6$ & $8 x_{1}+6 x_{2}+s_{2}=4$ \\
$x_{i}, \lambda_{i}, \mu_{i}, s_{i}, v_{i} \geq 0, i=1,2$ & $x_{i}, \lambda_{i}, \mu_{i}, s_{i}, v_{i} \geq 0, i=1,2$ \\
satisfying complementary conditions: & satisfying complementary conditions: \\
$\lambda_{1} s_{1}=0, \lambda_{2} s_{2}=0$, and $\mu_{1} x_{1}=0, \mu_{2} x_{2}=0$ & $\lambda_{1} s_{1}=0, \lambda_{2} s_{2}=0$, and $\mu_{1} x_{1}=0, \mu_{2} x_{2}=0$ \\
\hline Solution: $z_{S}=6.25, x_{1}=1,25$, and $x_{2}=0$. & Solution: $z_{I}=0.9, x_{1}=0.3$, and $x_{2}=0$ \\
\hline
\end{tabular}

According to Li and Tian [5], for the solution of the model in (11a)-(11c), the best optimum problem is $z_{S}=-0.9, x_{1}=0.3$, and $x_{2}=0$, the worst optimum problem is $z_{I}=-6.25, x_{1}=$ 1,25 , and $x_{2}=0$, and the optimum value is $\underline{z}=\left[z_{I}, z_{S}\right]=$ $[-6.25,-0.9]$.

This paper presents only the maximization problem so that any minimization problem will be converted into maximization problem, the simple procedure to convert a minimization problem to a maximization problem and vice versa. Simply multiply the objective function of a minimization problem by -1 converting it into a maximization problem and vice versa.

$$
\begin{aligned}
\text { Maximize } z & \\
= & {[6,10] x_{1}+[-3,-2] x_{2} } \\
& +[-1,1] x_{1} x_{2}+[-10,-4] x_{1}^{2} \\
& +[-20,-10] x_{2}^{2}
\end{aligned}
$$

subject to

$$
\begin{aligned}
{[1,2] x_{1}+3 x_{2} } & \leq[1,10] \\
{[-2,8] x_{1}+[4,6] x_{2} } & \leq[4,6] \\
x_{1}, x_{2} & \geq 0 .
\end{aligned}
$$

We apply the extension of Wolfe method for transforming quadratic programming with interval coefficients model in ((12a)-(12d)) into linear programming with interval coefficients model. We have

$$
\text { Minimize } z=v_{1}+v_{2}
$$

subject to

$$
\begin{aligned}
& {[8,20] x_{1}+[-1,1] x_{2}+[1,2] \lambda_{1}+[-2,8] \lambda_{2}-\mu_{1}} \\
& \quad+v_{1}=[6,10]
\end{aligned}
$$

$$
\begin{aligned}
& {[-1,1] x_{1}+[20,40] x_{2}+[3,3] \lambda_{1}+[4,6] \lambda_{2}-\mu_{2}} \\
& \quad+v_{2}=[-3,-2] \\
& {[1,2] x_{1}+3 x_{2} \leq[1,10]} \\
& {[-2,8] x_{1}+[4,6] x_{2} \leq[4,6]} \\
& x_{1}, x_{2} \geq 0 .
\end{aligned}
$$

We apply Algorithm 6 for transforming linear programming with interval coefficients model in ((13a)-(13f)) into two classic linear programming models with special characteristics, namely, the best optimum and the worst optimum problem. The result of the transformation is shown in Table 1.

So, the optimum value of the quadratic programming with interval coefficients is obtained by combining the optimum value from the worst and the best optimum problem; that is, $\underline{z}=\left[z_{I}, z_{S}\right]=[0.9,6.25]$. This solution gives the same value as obtained by Li and Tian [5].

\section{Conclusion}

This paper presents an extension of Wolfe method. The extension of Wolfe method performed by transforming the quadratic programming with interval coefficients model into linear programming with interval coefficients model. Furthermore, linear programming with interval coefficients model is transformed into two classic linear programming models using Algorithm 6. The extension of Wolfe method has a particular benefit: the final model is linear programming. Hence, it can be solved by the simplex method.

\section{Conflicts of Interest}

The authors declare that there are no conflicts of interest regarding the publication of this paper.

\section{References}

[1] F. S. Hillier and G. J. Lieberman, Introduction to operations research, Holden-Day, Inc., Oakland, Calif., Third edition, 1980. 
[2] P. Wolfe, "The simplex method for quadratic programming," Econometrica, vol. 27, pp. 382-398, 1959.

[3] R. E. Moore, Interval Analysis, Prentice-Hall, Englewood Cliffs, NJ, USA, 1966.

[4] S.-T. Liu and R.-T. Wang, "A numerical solution method to interval quadratic programming," Applied Mathematics and Computation, vol. 189, no. 2, pp. 1274-1281, 2007.

[5] W. Li and X. Tian, "Numerical solution method for general interval quadratic programming," Applied Mathematics and Computation, vol. 202, no. 2, pp. 589-595, 2008.

[6] S. C. Tong, "Interval number and fuzzy number linear programmings," Fuzzy Sets and Systems. An International Journal in Information Science and Engineering, vol. 66, no. 3, pp. 301306, 1994.

[7] K. Ramadan, Linear Programming with Interval [Msc. thesis], Carleton University, Ottawa, Ontario, 1997.

[8] J. W. Chinneck and K. Ramadan, "Linear programming with interval coefficients," Journal of the Operational Research Society, vol. 51, no. 2, pp. 209-220, 2000.

[9] D. Kuchta, "A modification of a solution concept of the linear programming problem with interval coefficients in the constraints," Central European Journal of Operations Research (CEJOR), vol. 16, no. 3, pp. 307-316, 2008.

[10] H. Suprajitno and I. B. Mohd, "Interval linear programming," in Proceedings of ICOMS-3, Bogor, Indonesia, 2008.

[11] G. Alefeld and J. Herzberger, Introduction to Interval Computations, Academic Press, New York, NY, USA, 1983.

[12] E. Hansen, Global optimization using interval analysis, vol. 165 of Monographs and Textbooks in Pure and Applied Mathematics, Marcel Dekker, Inc., New York, 1992.

[13] J. Zhang, "Optimality condition and wolfe duality for invex interval-valued nonlinear programming problems," Journal of Applied Mathematics, vol. 2013, Article ID 641345, 2013.

[14] H.-C. Wu, “The Karush-KUHn-Tucker optimality conditions in an optimization problem with interval-valued objective function," European Journal of Operational Research, vol. 176, no. 1, pp. 46-59, 2007. 


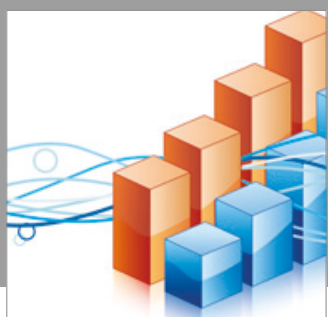

Advances in

Operations Research

vatersals

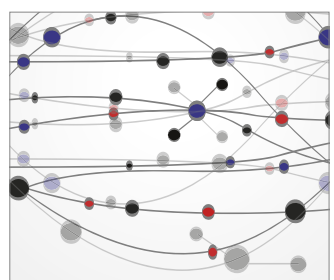

\section{The Scientific} World Journal
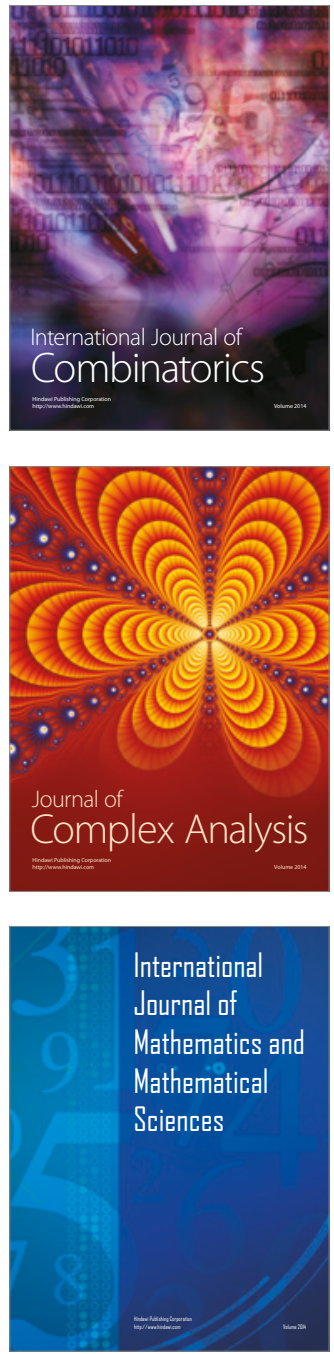
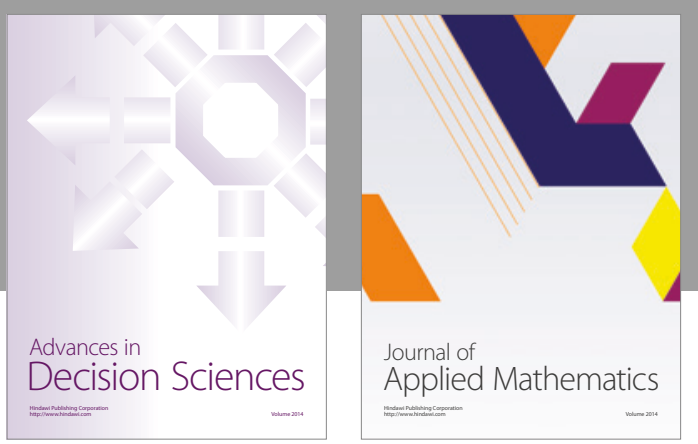

Algebra

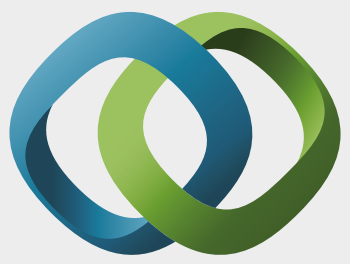

\section{Hindawi}

Submit your manuscripts at

https://www.hindawi.com
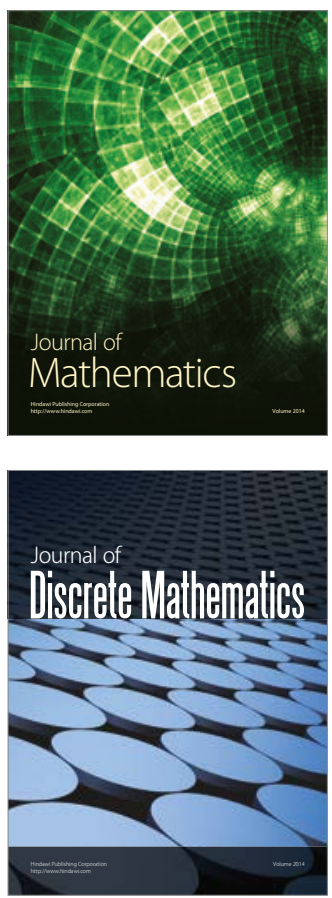

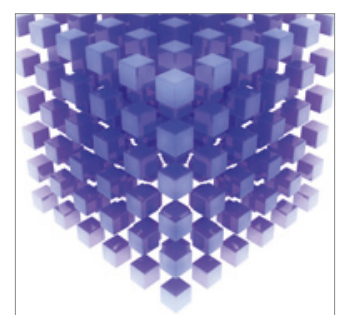

Mathematical Problems in Engineering
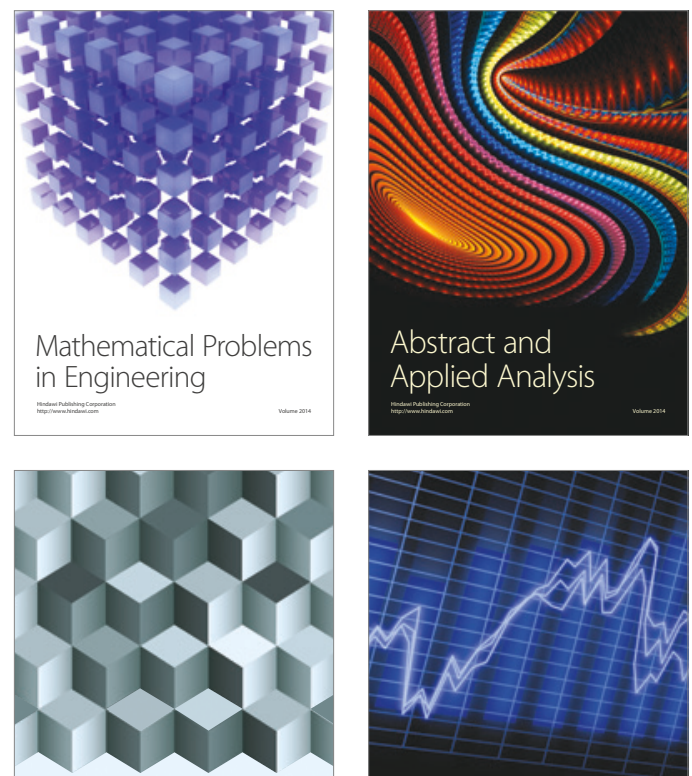

Journal of

Function Spaces

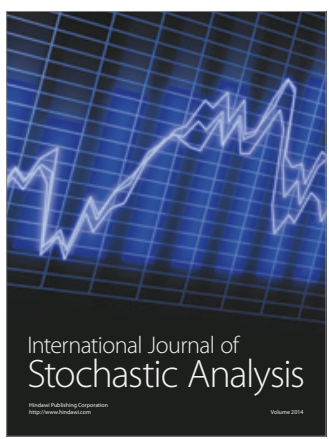

Probability and Statistics
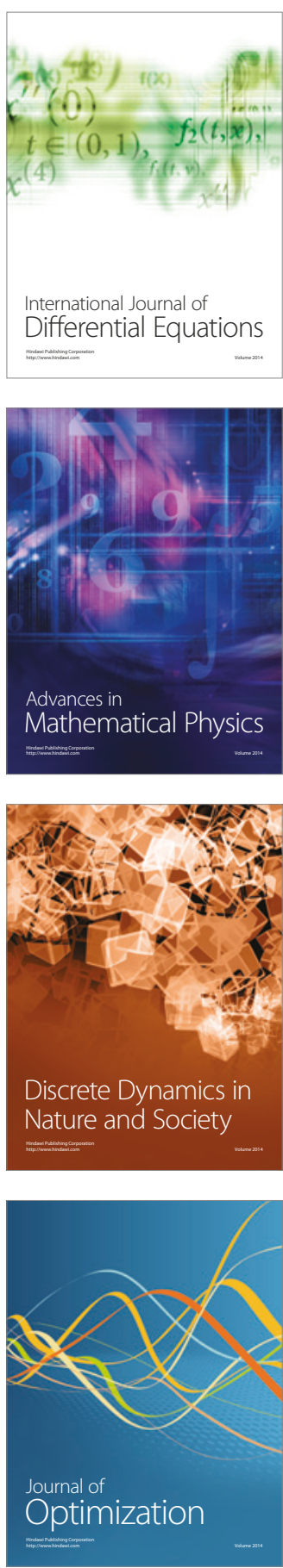